\title{
L'ESPAGNE, LA COMMUNE ET L'INTERNATIONALE
}

Les événements qui se produisirent en France lors du printemps de 1871 eurent une triple répercussion sur la situation en Espagne. Ils constituèrent en premier lieu la pierre de touche des tensions existant entre les groupes politiques dressés contre la Reine Isabelle II en 1868. Ils encouragèrent ensuite une réaction de plus en plus violente des groupes sociaux au pouvoir contre les organisations ouvrières de l'A.I.T. surtout, qu'on assimilait purement et simplement aux Communards. Ils constituèrent enfin, du fait même de cette réaction d'une part, par le truchement des émigrations provoquées par les persécutions déclenchées contre l'Internationale et son idéologie d'autre part, l'un des facteurs essentiels de la propagation de cette idéologie. C'est à l'étude détaillée de cette triple répercussion de la Commune sur la situation espagnole aux environs de 1870 que nous allons procéder.

LA COMMUNE ET LES TENSIONS POLITICO-SOCIALES DE 1871 EN ESPAGNE

La révolution de septembre 1868 avait coûté son trône à Isabelle II. Elle avait également uni en un vaste front placé sous une même bannière démocratique, celle de la lutte pour une égalité de droits authentique et pour un suffrage universel, tout un éventail de groupements politiques. Ces derniers comprenaient en effet aussi bien ceux que la politique suivie par la Reine Isabelle avait mis à l'écart au fur et à mesure de son évolution vers un conservatisme modéré, voire carrément réactionnaire, que ceux qui représentaient les nouvelles tendances de gauche, démocrates et républicains de type fédéraliste. Ces milieux politiques opposés à la Reine reçurent un puissant appui de la part des masses. Celles-ci étaient restées jusqu'alors plus ou moins étrangères à la politique. Elles cessent à ce moment de n'être qu'un instrument passif utilisé par n'importe qui dans son propre intérêt et se lancent, mues désormais par une authentique conscience de classe, à la recherche des voies politiques qui devaient leur permettre de satisfaire au 
mieux leurs aspirations économiques et sociales. Cependant les divergences entre les divers groupes qui avaient participé au soulèvement de septembre ne purent être aplanies que de façon provisoire, tant dans le domaine politique que social. Ils ne s'entendaient que sur un point, celui de l'opposition à l'ancien régime.

L'union dans la lutte révolutionnaire commença à se fissurer dès les lendemains de la victoire. Les mesures d'ordre prises, lors de sa mise en place à Madrid, par le gouvernement du Général Serrano provoquèrent des tensions entre les paysans d'Andalousie et d'Estrémadure d'une part, et le nouveau régime d'autre part. Il y eut lutte à main armée. Lorsque l'Assemblée Constituante du parlement (des Cortes) se réunit en mars 1869 , la gauche qui y était représentée, les républicains fédéralistes, engagea la bataille contre la nouvelle monarchie. Elle revendiqua l'abolition du service militaire promise lors de la campagne qui avait précédé le soulèvement militaire, promesse qui avait largement contribué à entraîner l'adhésion des masses populaires. Ayant perdu la bataille au parlement, les leaders républicains tournèrent alors leur propagande contre l'œuvre de l'Assemblée Constituante vers cette poudrière que constituaient les milieux ouvriers. Le résultat en fut l'explosion d'un vaste mouvement en Catalogne qui s'étendit à d'autres zones du pays; il fut d'ailleurs immédiatement condamné par les dirigeants fédéralistes eux-mêmes. Ceux-ci rappelèrent, un peu tard, qu'il convenait de suivre les voies légales. Ces événements avaient ouvert une brèche dans la foi que portaient les masses à la cause républicaine. Ils encouragèrent au cours des dernières semaines de 1869 et au début de 1870 , le développement des petits noyaux de l'Internationale créés à Madrid et à Barcelone par Fanelli, l'un des disciples et amis de Bakounine. Sur la proposition du noyau de Madrid, un Congrès des associations ouvrières eut lieu à Barcelone en juin 1870. Celui-ci donna une structure à la Región d'Espagne et à son Conseil fédéral.

Il convient de souligner ici deux faits. En premier lieu celui-ci que tant le Congrès que le mouvement ouvrier qui s'était développé dans le même temps, avec un nombre croissant de fédérations locales, ne se heurtèrent de prime abord à aucune barrière, à aucun obstacle de la part des autorités officielles. La législation démocratique qui venait d'être inaugurée garantissait tous les droits individuels, notamment ceux de réunion et d'association. Elle favorisait donc la tendance de l'Internationale. Les républicains fédéralistes, inconscients encore qu'ils étaient des brèches que l'organisation ouvrière allait ouvrir dans leurs propres rangs, eurent en effet au début un préjugé favorable à l'égard de celle-ci. En second lieu le fait que le mouvement socialiste espagnol revêtit dès l'origine un caractère totalement apolitique. Fanelli commit l'erreur de confondre les statuts, les principes, les 
tendances du mouvement internationaliste espagnol qui se développait avec ceux de l'Alliance de la Démocratie Socialiste fondée par Bakounine. Le Congrès de Barcelone recommanda à toutes les sections de l'Association Internationale des Travailleurs de s'abstenir de réaliser "quelque activité de groupe que ce soit ayant pour objet une transformation sociale basée sur les réformes politiques nationales». Les premiers mois de l'existence de la Fédération Régionale d'Espagne se caractérisèrent par un certain état de confusion aussi bien chez les républicains, pourtant convaincus que le "prolétariat militant" pouvait rentrer dans le cadre de leurs propres buts et de leurs propres programmes, que chez de nombreux néophytes de l'anarchie, lesquels ne se rendaient point encore compte que leur credo internationaliste les éloignait irrémédiablement des rangs républicains.

Dans de telles conditions, les événements de Paris eurent sur la situation espagnole une répercussion qui fut double et contradictoire. Ils eurent pour effet en premier lieu de mettre en garde la monarchie d'Amédée Ier, qui venait d'inaugurer son existence éphémère, contre l'organisation internationale. Les libertés individuelles garanties par la Constitution plaçaient jusques là cette dernière, comme il a déjà été dit, dans la légalité la plus absolue. Mais on chercha dès lors dans la Constitution elle-même les arguments nécessaires pour réagir, d'une façon qui fut de plus en plus rude, contre les Internationaux, considérés désormais comme constituant un grave danger pour l'Etat. $^{1}$ En second lieu, la polémique qui venait de commencer sur les buts et les dangers présentés par le mouvement communard contribua à réunir républicains fédéralistes et anarchistes espagnols. Le parti républicain fédéraliste se chargea de la propagande en faveur de la révolution parisienne, et de l'expliquer tant dans la presse qu'au parlement. Les républicains et les anarchistes mirent leur point d'honneur à s'identifier aux Communards français et se virent dans l'obligation de mettre leur organisation à l'abri des persécutions officielles. Les républicains espagnols voyaient dans le retentissant soulèvement parisien et dans la forme de gouvernement qu'il esquissa une justification et un triomphe de l'idéologie fédéraliste, par opposition aux traditions centralisatrices de l'ancien régime. Les membres de l'Internationale ne voyaient par contre que son aspect social, et le fédéralisme auquel ils aspiraient était bien différent de celui de Proudhon: c'était celui de Bakounine, que le Congrès de Barcelone avait repris à son compte.

C'est naturellement en Catalogne, région frontalière qui possède une

1 L'article 19 de la Constitution de 1869 stipule très opportunément que "Toute association dont l'objet ou les moyens compromettraient la sécurité de l'Etat pourra être dissoute par la voie d'une loi." 
longue tradition de tensions entre ouvriers et patrons ainsi qu'un ancien fond de revendications décentralisatrices, que les premiers symptômes d'agitation provoqués par la révolution de France se firent sentir. Il en était d'ailleurs toujours allé ainsi depuis 1789. En avril, alors que la bataille atteignait son apogée à Paris, Iglesias, gouverneur civil de Catalogne, commença à limiter, puis à interdire les réunions d'ouvriers. Allant plus loin encore, il voulut éviter que les troubles de Paris ne se reproduisent à Barcelone. Il autorisa donc le gouverneur militaire à former un contingent de troupes armées dans la Casa de Caridad, vaste édifice sis stratégiquement en plein cœur du quartier ouvrier le plus peuplé et le plus agité de la ville; il procéda également à de premières arrestations d'étrangers considérés "suspects". Ces faits provoquèrent le 22 mai au parlement une interpellation du député ouvrier Lostau au ministre de l'Intérieur, Sagasta. Les deux questions essentielles, à savoir la légalité des associations ouvrières et les répercussions de la Commune en Espagne, se trouvèrent pour la première fois mêlées dans l'interpellation du député et dans la réponse du ministre. Lostau énuméra les vexations commises par les autorités civiles et militaires, vexations auxquelles le comble fut mis par l'occupation de la Casa de Caridad et par l'arrestation de trois citoyens français, arrestations d'ailleurs faites contre le gré de la Députation provinciale de Barcelone: "Nous demandons [...] que le droit d'association soit librement exercé. L'attitude des membres de l'Internationale n'est point ambiguë. J'en fais partie et déclare que nous ne nous éloignons en rien de la voie de la justice et de la morale. Je ne fais donc que remplir un devoir vis-à-vis de mes concitoyens. Je termine en affirmant que si l'on ne respectait pas la liberté de réunion et d'association, les ouvriers sauraient également remplir leur devoir, même s'il était fait appel à la force brutale de l'armée."

Sagasta entama sa réplique par la lecture d'un télégramme qui venait d'être expédié par le gouverneur civil de Barcelone et dans lequel il faisait savoir que les usines Batlló venaient de reprendre leur travail après trois mois d'arrêt. Les autorités de Barcelone s'étaient bornées, dit le ministre, à dissoudre des réunions qui ne tendaient qu'à provoquer artificiellement des grèves injustifiées, et à défendre le droit des "ouvriers honnêtes» à exercer librement leur travail. Puis il releva en passant l'infiltration d'agents étrangers qui avaient pris part à ces conflits. Il attribua à la filiale espagnole de l'Internationale le caractère d'organisation subversive dirigée de l'étranger.

"C'est à Barcelone - dit-il - que le mal s'est présenté avec le plus de gravité, car parmi ces agents il y avait trois Français. A Barcelone, plus de 8.000 Français vivent à l'abri de nos lois. Ainsi donc, 
ces trois Internationaux ont été dénoncés, non seulement par des personnes de toutes sortes, mais encore par beaucoup de leurs dignes compatriotes qui ont intérêt à ce qu'on n'abuse pas de l'hospitalité. Ces Français ne portaient sur eux aucune pièce d'identité et n'exerçaient d'autre métier que celui d'agiter les masses ouvrières, de les exciter à la rébellion et de provoquer des grèves $[\ldots]$ Il faut dire à ces messieurs: vous n'avez rien à faire ici, et ceux de la Commune qui vous accréditent ne nous servent en rien $[. .$.$] Les ouvriers sont dans leur droit en demandant le salaire$ qui leur plaît, et dans leur droit les chefs d'entreprises en accédant à cette revendication ou en la refusant. Ce qui est criminel, c'est de promouvoir artificiellement les grèves, de détourner du travail les ouvriers honnêtes, et de perturber la société.»

Le discours de Sagasta laisse percevoir un net infléchissement dans la voie suivie par la "révolution démocratique» espagnole commencée en septembre 1868. Il y avait là déjà un peu comme un reflet de l'attitude de l'assemblée de Versailles à l'égard de la municipalité de Paris. L'allusion faite à la colonie française de Barcelone, plus alarmée encore que les Espagnols eux-mêmes de l'infiltration communaliste dans la péninsule, est fort significative. Il est d'ailleurs évident que Sagasta était fort méfiant, car, ancien conspirateur progressiste, il était connaisseur en matière de techniques subversives. Les Français arrêtés étaient loin d'être, comme le souligna Lostau, des suspects dépourvus de pièces d'identité. L'un d'eux était le correspondant de presse de l'Emancipation de Toulouse, et l'autre le correspondant d'un journal de Barcelone, et ni l'un ni l'autre ne faisaient partie de l'Internationale. ${ }^{1}$ Le troisième n'en était pas encore.

Lostau n'était pas seul face à la «réaction»: l'opinion publique républicaine fit cause commune avec lui. Dès les débuts des événements de Paris, le journal La Igualdad, organe madrilène des républicains fédéralistes, s'était posé en défenseur enthousiaste de la Commune. ${ }^{2}$ On ne trouverait guère de cas semblable dans toute la presse européenne de l'époque. Alors que la mise en cause de l'ordre dans la capitale française

1 Les personnes arrêtées étaient André Michel, journaliste, Royannez, ancien directeur de L'Emancipation de Toulouse, et Nodot, commerçant établi à Barcelone depuis cinq ans.

2 Il écrivait le 23 mars: "Ce que veut Paris, ce sont des garanties, des armes et une organisation, avoir l'assurance que l'Assemblée n'excédera pas ses pouvoirs, qu'il ne pourra jamais être attenté à l'existence de la République et aux libertés du peuple..." ("Lo que quiere París»). Il proclamait le 26, pour souligner le caractère lógal des événements, que "le sort de la liberté, l'avenir de la République et l'honneur de la France étaient totalement dissociés des hommes funestes de Versailles..." ("París y la Asamblea de Versalles») 
alarmait de plus en plus les divers Etats d'Europe, La Igualdad déclarait, le 26 avril, dans un élan d'enthousiasme fervent:

"Plus de militarisme ni d'entraves à l'initiative individuelle à laquelle le prolétariat doit son servage, et la patrie ses désastres et malheurs: telle est l'aspiration de la capitale de la France [...] Honneur et gratitude éternels à nos frères de Paris, qui combattent pour le triomphe de la République fédérative, qui selon les mots éloquents de la Commune, <est la fin du vieux monde gouvernemental et clérical, du militarisme, du fonctionnarisme, de l'exploitation, de l'agiotage, des privilèges ; c'est-à-dire de tous ces moyens d'exploitation du peuple par la classe moyenne, qui ont rendu impossible jusqu'à maintenant en Europe l'avènement de la démocratie et la libre et sûre jouissance de la vraie liberté.»

\section{II}

\section{LA REACTION DES MILIEUX GOUVERNEMENTAUX CONTRE LA COMMUNE}

Cette ardente défense du fédéralisme français par le fédéralisme espagnol a poussé le gouvernement de Serrano et de Sagasta à développer plus encore les mesures de sécurité préventives prises à Barcelone. Le 2 mai une réunion pacifique et pacifiste des partisans de l'association ouvrière eut lieu au Café internacional, établissement situé en plein cœur de Madrid. Elle se voulait une réplique aux manifestations patriotiques du 2 mai commémorant le soulèvement de 1808 contre les armées napoléoniennes. La réunion n'avait rien d'illégal. Elle fut cependant interrompue par la «bande de la matraque» (partida de la porra), qui était plus ou moins appuyée sous cape par le ministre de l'Intérieur. Le fait suscita de nouvelles protestations de la part des républicains, orchestrées, quoique sans grande vigueur, par certains secteurs de l'opinion peu favorables au ministre.

La presse «bien pensante», de son côté, a en général vu avec satisfaction cette réplique brutale à l' "Internationale apatride». La version des événements de Madrid donnée par le Diario de Barcelona, journal modéré et bourgeois, est significative. "L'audace de cette société [l'Internationale] n'en est-elle pas arrivée dernièrement au point de vouloir protester publiquement contre l'anniversaire du 2 mai, dont l'heure arrivait, de l'interdire de par sa propre volonté ou son influence; à la suite de quoi viendrait la démolition du monument qui rappelle au peuple espagnol le fait le plus glorieux de son histoire provoqué par la félonie et la trahison d'une armée étrangère? Nous ne doutons pas que si une telle chose se produisait, le peuple de Madrid ne contemplerait pas impassible la destruction de l'obélisque du 2 mai, 
élevée sur les cadavres et le sang de ses pères, comme le peuple de Paris se dispose à voir tomber, sans manifester la moindre émotion, la colonne de la Grande Armée, fruit sanglant de la victoire de ses soldats. Nous croyons encore le peuple espagnol suffisamment digne pour interdire qu'aucun pouvoir, même invoquant la théorie la plus brillante ou la plus insensée, ne détruise les monuments qui représentent son héroïsme et son amour patriotique."

Le contraste qui apparaît de la dialectique astucieuse de La Igualdad dans ce jeu de symboles architectoniques, ne laisse pas d'être curieux. Ce journal, qui avait jeté les hauts cris à propos du coup de force perpétré le 2 mai par des agents camouflés du gouvernement, a en effet déclaré, tant pour justifier la destruction de la colonne Vendôme que la "raison d'être" de l'obélisque de Madrid, ce qui suit: "La colonne de la place Vendôme a dû disparaître; et si la Commune de Paris l'a fait abattre, elle a bien fait; si elle ne l'a pas encore fait, elle fera bien en le faisant. Les Français doivent seulement conserver la colonne qui rappelle le triomphe de la Bastille, c'est-à-dire la fin de l'ancien régime, et nous le monument du 2 mai, parce que ces monuments rappellent tous deux la résistance à l'oppression. Dans l'Europe fédérale il n'y aura plus par la suite de peuples ennemis..."

Dans les jours qui suivirent, les violences incontrôlées de la fin de la révolution communale, les incendies de Paris et l'exécution des otages, furent sévèrement condamnées aux quatre coins de cette Europe qui demeurait pourtant insensible devant la dureté de la répression versaillaise. Lorsque sa voix se fut éteinte, la Commune continua à trouver à Madrid un défenseur décidé dans La Igualdad. Ce journal continua à proclamer l'excellence du mouvement révolutionnaire tout en déplorant ses excès. Il estimait en effet qu'il ne fallait pas imputer ces excès à la Commune de Paris, ses membres «n'ayant plus la moindre autorité depuis que l'insurrection avait été jugulée par d'autres hommes". L'attitude du journal républicain déchaîna l'indignation des organes ministériels, El Imparcial et La Iberia. Le dernier jour de la Commune, le 28 mai, La Igualdad répliqua à ses détracteurs et publia un article dont on devait peu après longuement débattre au parlement.

«Le programme de la Commune, les buts de la révolution de Paris, les droits du peuple parisien, voilà ce que La Igualdad a soutenu et défendu, mais jamais tous les actes des hommes qui ont pu prendre une part active dans l'insurrection. Si les révolutions et les partis politiques devaient être jugés sur les attentats et les crimes qu'auraient pu commettre beaucoup de leurs partisans, nul homme honnête et généreux ne pourrait tolérer maintenant le nom de ceux-là même qui se trouvent au pouvoir [...] Tous les partis, en mettant 
en œuvre leur idéal politique, ont le malheur de rencontrer soit des insensés fanatiques soit d'exécrables scélérats qui, dans l'ombre de son drapeau, travaillent à en souiller les principes. Que le parti qui se sent libre nous jette la première pierre."

Deux jours auparavant, le Conseil des ministres s'était occupé, à la suite des requêtes venues de Versailles et notamment de la circulaire de Jules Favre, du problème des émigrés français impliqués dans la Commune. Ln de ses porte-parole fit savoir le 27 mai qu'on prendrait «les mesures nécessaires pour empêcher les vandales de Paris de se réfugier en Espagne et de se soustraire de la sorte au châtiment que méritaient leurs crimes; qu'on créerait, semble-t-il, un dépôt à la frontière où l'on arrêterait les personnes qu'on entend emprisonner pour les livrer ensuite aux autorités françaises en tant que prisonniers de droit commun concernés par les traités d'extradition ». ${ }^{1}$ Cette déclaration officieuse a donné lieu les 29 et 30 mai à un intéressant débat parlementaire. Ce fut le moment culminant où l'on put voir l'impact des événements de la Commune sur la politique espagnole; il fut aussi à l'origine de la décision prise quelques mois plus tard au sujet de la légalité ou non de l'association internationale. Oriol Vergés y a récemment consacré une étude qui, bien qu'étroitement en rapport avec celle-ci, ne parle point du problème des émigrés ni du jugement concret porté sur la révolution parisienne.

Sagasta se borna, le 29, à définir l'attitude du gouvernement au sujet des réfugiés de la Commune. Il suivit grosso modo l'exemple fourni par la Belgique, autre pays limitrophe de la France. Les émigrés devaient être livrés aux autorités françaises conformément aux traités d'extradition. Mais les termes qu'employait Sagasta ne faisaient pas de différence entre les personnes poursuivies du fait de leurs idées, et celles poursuivies du fait d'actes délictueux. L'opposition républicaine prépara immédiatement une motion de censure contre cette déclaration. Le grand orateur Castelar craignit que cette initiative des amis de l'Internationale n'eût un effet contraire à celui qu'on souhaitait, du fait du climat qui régnait alors au parlement et dans le pays. Il prit habilement les devants et interpella le premier le ministre des Affaires étrangères, Martos, lui demandant de faire savoir si les extraditions prévues n'allaient pas ôter de leur efficacité aux mesures d'entente prises entre la France et la Belgique au sujet des émigrés politiques.

La réponse fournie par Martos fut fort précise et mesurée. Elle

1 On relève déjà dans les numéros des 29 et 30 mai du Diario de Barcelona des informations sur des arrestations diverses d'émigrés français. 
établit un distinguo sans ambages qui répondait aux désirs de Castelar, mais n'était pas tout à fait conforme aux points de vue de Sagasta:

«Les portes de la patrie espagnole ont toujours été, et seront toujours, ouvertes [...] à ceux qui venaient les franchir poursuivis pour leurs opinions politiques [...] Le Gouvernement espagnol prendra en considération les réclamations dûment faites du Gouvernement français, bien que sur ce point le Gouvernement espagnol ait sa loi qui est le traité, et le traité en vigueur sera observé; quand on lui demandera l'arrestation dûment fondée d'un criminel $[\ldots]$ il appréciera comme il le doit les motifs en vertu desquels cette arrestation lui est demandée, il décrétera d'arrestation et gardera en arrestation la personne qui est l'objet de cet acte, la tenant à la disposition des agents du Gouvernement français; ceux-ci dans le délai convenable et dans le cadre du traité pourront demander, si bon leur semble, l'extradition de cette ou de ces personnes; et quand les requêtes seront formulées conformément au traité et justifiées dans le traité, le Gouvernement espagnol les examinera et [...] cette fois avec plaisir, parce qu'il contribuera à un acte de justice et d'humanité, s'empressera de remettre en bonne et due forme, en exécution du traité, de son devoir et de la loi, les personnes que réclame le Gouvernement français.»

La réponse tranquillisa Castelar, mais il rappela à Martos que «les traités exigeaient que l'arrêt d'incarcération délivré par le juge de la nation réclamant le prisonnier fasse l'objet d'un examen par les tribunaux et les autorités de la nation auprès de laquelle le prisonnier s'était réfugié». Il ne manqua point, en terminant son bref discours, de rappeler les circonstances qui avaient provoqué les délits dont il était maintenant question, vingt ans de césarisme et la disparition de la liberté.

Cette passe d'armes parlementaire déplut sur les bancs de la majorité de l'Assemblée, aussi bien qu'à tous les conservateurs en général. Il existait en effet une dangereuse divergence de points de vue entre Martos et Sagasta, malgré l'habileté du premier. Le Diario de Barcelona souligna qu'en affirmant que le gouvernement espagnol était d'accord pour livrer au gouvernement français les émigrés qui relevaient du droit commun, il ne faisait que s'en tenir à la légalité et aux traités en vigueur: qu'en l'occurrence le gouvernement français considérait a priori que tous ceux qui avaient pris part à l'insurrection de Paris relevaient du droit commun; que $M$. Sagasta se montrait d'accord avec ce point de vue, mais que M. Martos ne l'admettait point. Désireuse de combler le fossé et de porter un coup au succès remporté par Castelar, 
la majorité présenta le 30 du même mois une motion contre «les attentats commis par la Commune de Paris", espérant la faire approuver par le parlement, comme les autres parlements d'Europe l'avaient fait. ${ }^{1}$

Pí y Margall, alors le grand pontife du fédéralisme républicain, réagit immédiatement contre la confusion qui était faite délibérément entre les idées de la Commune et les excès que l'on entendait condamner. On peut relever un parallélisme évident entre les termes qu'il employa et ceux qu'avait utilisés La Igualdad dans ses éditoriaux du même moment.

4Je comprendrais - dit-il - que nous agissions avec cette passion, si nous appartenions à un pays où la révolution n'eût pas taché l'histoire de ses crimes; mais ici nous avons commis les plus grands crimes, ici nous avons vu en 34 les populaces insurgées pénétrer dans les couvents et égorger les moines; ici, nous avons vu la populace entrer dans les arsenaux et fusiller tous les prisonniers politiques qui s'y trouvaient sans épargner ceux qui étaient dans les hôpitaux; ici, nous avons vu fusiller un frère du général O'Donnell, nous l'avons vu traîner ensuite à travers les rues de la ville, lui couper la tête et la mettre au bout d'une pique. Nous avons commis ici toutes sortes d'assassinats. Durant la guerre civile nous avons expulsé des villages des familles entières pour avoir eu seulement un membre dans le camp ennemi; dans nos discordes civiles nous avons égorgé des villages [...] Ces souvenirs vous font mal, messieurs de la majorité? moi aussi, ils me font souffrir comme vous, mais je les rappelle pour que vous soyez justes et impartiaux quand il s'agit des crimes des autres peuples; car je vous en avertis: ces crimes ne sont pas fils de la méchanceté humaine, mais lorsque l'homme perd la raison, il cesse d'être un homme pour se transformer en bête fauve [...]"

Ni une réplique de Rios Rosas, ni celle de Sagasta ne parvinrent en fait à diminuer la force des arguments de Pí y Margall. Le ministre de l'Intérieur déplora "en tant qu'Espagnol» que l'Espagne soit le seul pays où l'on trouve unne presse qui excuse et approuve chaudement les horreurs de Paris», «un parti, le seul au monde, qui ose s'associer aux crimes de la Commune et lui envoyer des ambassadeurs pour la féliciter, alors que sa nature était déjà claire, alors que des faits d'ores et

1 «Le Congrès a entendu avec satisfaction les protestations énergiques faites par le Gouvernement contre les attentats horribles commis par la Commune de Paris. Il s'associe au sentiment d'indignation qu'éveille dans toutes les consciences la conduite des criminels qui ont violé les lois de l'humanité. "Signé: Luis Peñuelas, Fernando León, Gaspar Núñez de Arce, Antonio Palau, Angel Mansí, A. Merelles, Cándido Martínez. 
déjà consommés permettaient de prévoir ceux qui allaient l'être et apporter un comble à son exécrable façon de procéder».

García López, directeur de La Igualdad, se sentit visé et intervint une nouvelle fois pour établir une distinction entre les principes politiques de la Commune et les délits qui lui étaient imputés: "C'est pourquoi nous avons admiré cette grande révolution que personne à cette heure ne peut bien apprécier mais que les siècles futurs béniront peut-être, et ce serait de notre part une déloyauté et une méconnaissance totale de la justice, si nous approuvions la supposition gratuite des délits dont personne ne peut prouver que la Commune les a commis [...] Après que la Commune a disparu en tant qu'autorité publique, après qu'elle a envoyé à Versailles des émissaires en quête d'un arrangement, et qu'elle a déclaré qu'à partir de cet instant elle ne répondait plus de ce qui arriverait, des crimes ont eu lieu que nous déplorons tous, et dont vous ne savez même pas par qui ils ont été commis [...]"

Le vote sur la mention de censure fit apparaître au sein de la majorité républicaine, du fait de la scission apparente provoquée dans les rangs du gouvernement par le débat de la veille, une diversité de points de vue qui fit qu'elle ne rejeta point en bloc la formule de censure. Pí dut intervenir à nouveau; il s'exprima comme suit:

«La majorité va croire que ce qui est en train de se passer révèle une profonde division parmi nous. Non: le parti républicain ne peut se diviser parce qu'il est uni par une idée: la fédération ellemême. Nous, nous sommes avec la Commune parce que celle-ci voulait effacer l'ancienne tradition jacobine de la République une et indivisible, et ces principes nous les professons tous. En ceci il n'y a pas de division; s'il peut y en avoir une sur des questions de tactique, cela ne signifie rien. Quelques-uns ont cru que le vote pour la proposition qui a été discutée signifiait un assentiment à l'assertion que la municipalité de Paris avait commis des crimes, et pour cela, nous ne l'avons pas votée; si on avait censuré les crimes commis par quelques individus du peuple de Paris, nous aurions été disposés à les condamner. Comme il n'en est pas ainsi, nous attendons, messieurs, que la question puisse se décider non pas avec passion mais avec raison.»

La note finale au débat fut apportée le 31 par La Igualdad dans sa chronique parlementaire. Elle déclara ouvertement sur un ton prophétique:

"Le jour viendra où ceux qui ont formé la Commune de Paris pourront parler; le moment de les écouter et de les connaître viendra; alors l'opinion publique en France et en Espagne, en 
Italie et en Belgique, en Angleterre et en Prusse et partout, se modifiera: aujourd'hui, le jugement sur la Commune est abâtardi dans tous les pays [...]"

Elle soutint au sujet de la motion de censure de la veille, faisant écho aux paroles de $\mathrm{Pi}$, que "ce qui aurait été digne, noble et juste pour une assemblée de législateurs espagnols, aurait été de lancer l'anathème contre tous les crimes commis au détriment de la civilisation par les deux partis dans la lutte titanesque de Paris contre Versailles..."1

Le fédéralisme républicain continua donc à glorifier la Commune morte, y voyant le symbole d'une révolution politique manquée mais inévitable. Le fédéralisme anarchiste publia de son côté, quatre jours plus tard, un vaste programme intitulé "Manifeste envoyé par quelques partisans de la Commune à tous les pouvoirs de la terre». ${ }^{2}$ Il était signé par Anselmo Lorenzo, Francisco Mora et Tomás González Morago, et constituait un nouveau et violent rejet de la "révolution politique» au nom de la «révolution sociale». Ce document refit l'historique de la longue période au cours de laquelle le peuple, avide de justice, n'avait été qu'un instrument inconscient au service d'idéaux qui n'étaient point les siens. La Commune avait ouvert une nouvelle voie: "Mais comme ceci ne faisait l'affaire d'aucune des fractions en lesquelles se subdivise la classe moyenne pour se disputer la possession des privilèges, tous les partis politiques, les plus éminents chefs du parti républicain, le gouvernement de Versailles, la classe moyenne de France, les gouvernements de l'Europe, la classe moyenne de l'Europe et la presse, qui était toute à son service, les uns avec plus de franchise, les autres avec plus d'ambiguité, sans doute parce qu'ainsi ils servaient leur jeu, tous se sont coalisés contre le mouvement et les hommes de Paris $[\ldots] "$

Ces termes pouvaient être considérés comme un geste de mépris à l'égard des républicains fédéralistes qui avaient défendu "leur notion» de la Commune au parlement et dans la presse. Ils coupaient tous les ponts. Ils constituaient un véritable défi. Les membres espagnols de l'Internationale disent encore, sans établir la moindre distinction entre l'idée et les excès, excès que Pí et ses partisans avaient attribués à la folie provoquée par le désespoir:

1 Il convient de souligner que tant l'intervention parlementaire de Pí y Margall que celle, et plus encore, de García López, ont immédiatement provoqué l'envoi de messages d'adhésion de la part d'un grand nombre de clubs et comités républicains de toute l'Espagne. La Igualdad les publia à partir du 31 mai.

${ }^{2}$ Je cite d'après une photocopie en ma possession de ce document extrêmement rare. 
«Les événements de Paris, que, pour ce qui se réfère à la Commune, nous acceptons dans leur totalité, sans adroite exception d'aucune espèce, sont venus nous prouver que si un jour vous nous traîniez dans la lutte de classe, si eux ont brûlé, si eux ont fusillé et assassiné, nous, nous devrons réduire ces trois extrêmes en un seul. Nous, nous sauterons avec les villes et avec vous."

Le ton extrêmement violent de cette proclamation constituait une réplique au fléchissement conservateur qu'on a relevé dans les termes employés par Sagasta le 29 mai. La rupture entre l'Etat bourgeois et l'organisation ouvrière est devenue fait accompli au cours de la semaine cruciale du 29 mai au 4 juin. A compter de cette date, il ne s'est plus agi d'un dialogue ou d'une intégration; on était dans un cercle vicieux dans lequel le gouvernement essayait de trouver des arguments pour déclarer la guerre à l'Internationale en s'appuyant sur l'exemple de Paris, les porte-parole de celle-ci prenant plaisir à les lui fournir et à se signaler comme constituant un danger pour lui.

Ce qui en mai n'avait constitué qu'une déclaration concrète au sujet de la Commune et de ses partisans s'est en effet transformé quelques mois plus tard, en octobre et novembre 1871, en une véritable offensive parlementaire contre l'Internationale, lors du fameux débat étudié en détail par le professeur Vergés. ${ }^{1}$ L'opposition républicaine, Pí et Salmerón surtout, ont défendu encore contre l'opinion du gouvernement et de la majorité, le respect des droits individuels garantis par la Constitution, la liberté de réunion, d'association et d'opinion. ${ }^{2}$ Il existait néanmoins une grande différence entre cette position et celle d'une véritable "ouverture" à la révolution sociale de l'Internationale. Ainsi que l'a écrit Vergés, «l'écrasante majorité obtenue le 10 novembre 1871 au parlement ne laisse subsister aucun doute sur un point précis, à savoir que l'Association Internationale des Travailleurs était considérée comme immorale et dangereuse tant pour l'ordre public que pour la sécurité intérieure par les partis du centre et de droite. Elle demeurait donc en marge de la Constitution de 1869 et le gouvernement se voyait conférer les pouvoirs nécessaires pour procéder à sa dissolution. La liberté d'association ayant été proclamée, la question était délicate et pouvait être interprétée diversement selon l'idée que l'on se faisait des droits individuels. Les républicains croyaient que ces principes étaient absolus et hors de portée de la loi. Ms n'y voyaient donc aucun interdit

1 Oriol Vergés Mundó, La Primera Internacional en las Cortes de 1871, Publications de la Chaire d'Histoire de l'Espagne, Faculté des Lettres de l'Université de Barcelone, 1964.

${ }^{2}$ Cf. parmi les documents annexes à l'ouvrage cité les discours de Salmerón et Castelar. 
pour l'A.I.T. Les hommes influents du parti le plus radical de la Chambre n'étaient cependant pas d'accord avec les idées fondamentales de l'Internationale [...] C'est ainsi que Pí y Margall s'est déclaré opposé à la collectivisation et à la disparation de l'Etat juridique et organique. Les républicains sont paradoxalement devenus les défenseurs de ceux qui les avaient jadis répudiés, utilisant pour ce faire la presse. Il convient de voir dans ces interventions parlementaires un effort de plus réalisé par le parti fédéral pour attirer à lui le noyau internationaliste ouvrier." ${ }^{1}$

Lorsqu'en 1873 cette gauche bourgeoise représenta l'Etat au sein de l'éphémère première République, les faits ne laissent aucun doute à ce sujet. Mais le "prolétariat militant" avait déjà indiqué sans ambages lors de son assemblée clandestine de septembre 1871 à Valence les divergences insurmontables qui existaient entre eux.

"Si l'on considère que le vrai sens du mot République, en latin Res publica, signifie chose publique, chose propre à la collectivité, ou propriété collective, que démocratique est la dérivation de démocratie, qui signifie le libre exercice des droits individuels, lequel ne peut se rencontrer que dans l'anarchie $[\ldots]$; si l'on considère les droits de l'homme comme imprescriptibles et inaliénables, on en déduit que la Fédération doit être purement et exclusivement économique. La conférence des délégués espagnols de la Región espagnole de l'Association Internationale des Travailleurs, réunie à Valence, déclare: que la vraie République démocratique fédérale est la propriété collective, l'Anarchie et la Fédération économique, c'est-à-dire, la fédération libre et universelle des libres associations d'ouvriers agricoles et industriels, formule qu'elle accepte en totalité.»" ${ }^{2}$

III

\section{LA PROPAGATION ET LE RENFORCEMENT \\ DE L'INTERNATIONALE ANARCHISTE LA COMMUNE ESPAGNOLE}

Avant que cette "Conférence de Valence» ne se réunisse, entre les mois de juin et septembre 1871, l'Internationale connut en Espagne une première crise dont elle sortit renforcée. Le 3 juin, jour où sa procla-

1 Op. cit., p. 87.

2 Organización social de las secciones obreras de la Federación Regional Española, adoptada por el Congreso Obrero de Barcelona en junio de 1870 , reformada por la Conferencia regional de Valencia celebrada en septiembre de 1871 , y recomendada por el Congreso de Zaragoza, celebrado en abril de $1872,2^{\mathrm{e}}$ éd., Valence 1872, pp. 51-54. 
mation virulente en faveur de la Commune fut rédigée, le Conseil fédéral décida, convaincu qu'il était que le gouvernement allait déchaîner une persécution sur une grande échelle contre les fédérations locales, puisque celle de Barcelone avait été liquidée par la police, de s'exiler au Portugal. Mais il fut décidé upour tourner l'article de la Constitution interdisant toute société dont la direction se trouvait hors d'Espagne, de ne rien dire aux fédérations locales au sujet de notre siège, de faire acheminer leur correspondance à Madrid, d'envoyer la nôtre de cette ville, d'établir à cette fin Angel Mora à Madrid en feignant pour y parvenir d'accepter sa démission de membre du Conseil fédéral....11

Morago, Lorenzo et Francisco Mora arrivèrent le 7 à Lisbonne et y demeurèrent trois mois. Mais ce fait constituait un "impact indirect" de la Commune sur la situation espagnole. Il eut des conséquences imprévues, telles que l'éclosion au Portugal de l'internationalisme, du fait de la propagande active menée par les réfugiés espagnols. L'implacable persécution qui était prévue n'alla pas d'ailleurs très loin. L'accès au pouvoir, à l'occasion d'un changement de Cabinet, de Ruiz Zorrilla, rival politique de Sagasta et personnalité très proche de la position bienveillante des républicains à l'égard de l'organisation ouvrière, mit fin à la répression à partir de juillet. Le Conseil fédéral revint en Espagne en septembre, mais peu après, une nouvelle crise ministérielle et le retour au pouvoir du groupe dépendant de Sagasta furent à l'origine d'une nouvelle offensive des autorités au pouvoir. Celle-ci se traduisit aux mois d'octobre et novembre par le débat au parlement dont on a parlé et par la condamnation de l'organisation ouvrière, désormais déclarée inconstitutionnelle. Le double problème que posait l'affaire des «crimes de la Commune» et le danger représenté par l'Association Internationale des Travailleurs apparut encore au cours des premiers mois de 1872 dans la circulaire que Sagasta fit parvenir aux divers gouvernements européens dans le but d'obtenir d'eux une réaction unanime. ${ }^{2}$

L'année 1872 fut une année d'épreuves, tant du fait de la dureté des persécutions menées contre les fédérations locales espagnoles (et malgré leur extraordinaire prolifération au cours des années 1872 et 1873), ${ }^{3}$ que

\footnotetext{
1 A.I.T., Actas de los Consejos y Comisión Federal de la Región Española (18701874) [Colección de documentos para el estudio de los movimientos obreros en España en la época contemporánea], Barcelone 1970, t. I, p. 63.

2 "A une action commune pour contrecarrer les travaux de l'Internationale." Cf. Annexe II de l'article de Carole Witzig.

${ }^{3}$ Cf. l'étude "Acotaciones documentales para el estudio de la Internacional en España" que j'ai publiée in: Actas de los Consejos y Comisión Federal de la Región Española (1870-1874), t. I (Introduction).
} 
plus encore du fait de la scission du front ouvrier en deux clans, celui des autoritaires et celui des libertarios, scission qui ne constituait qu'un reflet des brèches ouvertes sur une plus grande échelle lors des Congrès de La Haye et de Saint-Imier. Le Congrès régional de Cordoue suivit en décembre 1872 la ligne de conduite indiquée en 1870 à Barcelone et confirmée à Valence en septembre 1871. Il plaça résolument la Región d'Espagne dans la ligne de Bakounine et l'opposa, selon l'expression utilisée par les anarchistes espagnols pour désigner Karl Marx, au "grand sultan de Londres». L'éclosion partielle à Madrid d'un "socialisme autoritaire», organisé par un réfugié de la Commune, Paul Lafargue, ${ }^{1}$ n'a représenté en réalité qu'une minorité insignifiante. Ni ce groupe «autoritaire», ni les républicains fédéralistes ne sont d'ailleurs parvenus à trouver un terrain commun d'entente. L'Internationale de la péninsule ibérique continua à être anarchiste, et c'est cette semence venue d'Espagne qui atteignit les côtes d'Amérique au cours des mois suivants. Elles furent portées jusqu'à Buenos Aires ${ }^{2}$ par les vagues d'une véritable diaspora provoquée par les répressions menées du temps de Sagasta aussi bien que du temps de Salmerón et Castelar, en plein régime républicain, ces derniers ayant été alarmés par les événements d'Alcoy. ${ }^{3}$

C'est à Alcoy que les Espagnols virent confusément apparaître sous les apparences d'une rébellion cantonaliste prétendant implanter un "fédéralisme partant d'en bas" par opposition au fédéralisme "partant d'en haut» de Pí y Margall, le reflet ibérique de la légendaire révolution rouge de Paris. Ce qui avait constitué pour l'ardente propagande bourgeoise de la revue La Defensa de la Sociedad un épouvantail terrifiant, les violences des Communards de Paris, était désormais devenu un fait dans une authentique "version» ibérique de l'exemple français,

${ }^{1}$ L'arrivée en Espagne de Paul Lafargue eut en effet lieu en août 1871. Lafargue ne se trouvait pas à Paris, mais à Bordeaux. Personnalité de premier plan au sein de l'Internationale - il était, comme on sait, gendre de Marx -, il a su se mettre soigneusement à l'abri de l'autre côté de la frontière lorsque les persécutions contre la Commune et ses complices commencèrent en France. Il se trouvait déjà à Madrid en décembre et s'y efforça, avec les rédacteurs de La Emancipación, d'organiser un noyau internationaliste suivant les orientations du Conseil de Londres.

${ }^{2} \mathrm{Cf}$. en ce qui concerne la propagation de l'Internationale en Amérique par les réfugiés espagnols, mon étude déjà citée, pp. 54-58.

${ }^{3}$ Les événements d'Alcoy ont été provoqués par un mouvement de grève contre lequel le maire, Albors, avait essayé de réagir par la force. Les masses prirent la Mairie d'assaut. Albors fut assassiné et traîné dans les rues. La ville se trouva pendant un certain temps au pouvoir des éléments populaires rattachés à l'Internationale. 
ce que l'on appela le "délire rouge» d'Alcoy. ${ }^{1}$ Castelar joua alors dans l'Espagne de 1873 un rôle qui rappelle par sa dureté celui de Thiers, créateur et "sauveur» de la République bourgeoise française. Thiers avait dû faire face à un mouvement au sein duquel se trouvaient mêlées tant la révolution politique du fédéralisme radical que la révolution sociale de l'Internationale ouvrière. Castelar dut livrer bataille, au nom d'un "républicanisme de l'ordre" de type centraliste, à un cantonalisme subversif et au prolétariat militant.

Mais ces deux mouvements n'étaient point amalgamés en Espagne. Ils se contentèrent de s'associer l'un à l'autre. Il me semble opportun, pour terminer cette analyse des événements engendrés au sein de la révolution espagnole par la révolution parisienne, de reproduire un extrait de la lettre envoyée en septembre par les membres de la Commission fédérale de la Región d'Espagne à leurs collègues des Etats-Unis afin d'éviter des malentendus:

«Vous n'avez pu apprécier avec exactitude ce qui arrive en Espagne, parce que les journaux bourgeois le transforment et le falsifient complètement et les ouvriers qui recueillent des nouvelles dans ces organes de la bourgeoisie se heurtent au même défaut. Le mouvement d'Alcoy a été un mouvement purement ouvrier, socialiste révolutionnaire. Le mouvement de Carthagène est purement politique et bourgeois. Et cela tellement, qu'à Carthagène il existe un gouvernement en face du gouvernement de Madrid, comme le gouvernement carliste qui existe à Estella se trouve face à ce dernier. C'est dire qu'en Espagne, les amis de l'autorité n'ont pas à se plaindre d'un manque de gouvernements car si nous comptons bien nous en avons trois. A Carthagène il n'y avait pas d'Internationaux et nous doutons qu'aujourd'hui il en existe un qui prend la défense de ce canton, ce qui suffit pour démontrer que le mouvement d'Alcoy est bien différent de celui de Carthagène: le premier fut seulement une revendication des Internationaux qui voyaient leurs droits foulés aux pieds par le maire et l'autorité municipale, et Carthagène fut seulement un mouvement politique dans l'unique dessein de prendre le pouvoir et continuer à exploi-

${ }^{1}$ La Defensa de la Sociedad publia son premier numéro le premier avril 1872. Son titre complet était: La Defensa de la Sociedad, Revista de los intereses permanentes y fundamentales contra las doctrinas y tendencias de la Internacional, ajena por completo a todo partido político. Religión, familia, patria, trabajo y propiedad. Parmi ses collaborateurs figurait la majeure partie des hommes marquants du libéralisme, Cánovas, Moret, les représentants du traditionalisme légitimiste Aparisi y Guijarro, Nocedal et les "ultra-catholiques» dont Alejandro Pidal. Le journal de l'Internationale El Condenado signala sa publication dans un article débordant d'ironie. 
ter les classes laborieuses. Dans les soulèvements (cantonaux) de Valence, Séville, Cadix, Grenade, Xérès, Sanlúcar, San Fernando, Carmona, Lebrija, Paradas, et Chipiona, les Internationaux prirent une part active aux événements pour être ensuite abandonnés par les guignols politiques. A Séville et Valence, uniques agglomérations où il y eut lutte, on peut dire que les Internationaux furent les seuls à se battre." ${ }^{1}$

Traduit de l'espagnol

${ }^{1}$ La lettre reproduite est copiée à la main dans le Registre du Conseil Fédéral d'Espagne (Bibliothèque Arús, Barcelone). Je l'ai déjà publiée dans ma présentation du livre de José Termes, El movimiento obrero en España, Barcelone 1965. 